\title{
Review on a Node Scheduling based on Partition for WSN
}

\author{
Shivani Thakur \\ Student M.Tech, Maha Markandeshwar University \\ Mullana, India
}

\author{
Abhay Bindal \\ Asst. Professor, Maha Markandeshwar University \\ Mullana, India
}

\begin{abstract}
WSN can have a node scheduling based on partition. Employment of such technique can reduce energy consumption of wireless sensor nodes and prolong the network lifetime. Firstly the monitoring area was partitioned into equal virtual grid, according to which the algorithm has two working mode one is monitoring mode and other one is tracking mode. In case of former only one node worked in each grid according to scheduling cycle for the reduction of energy consumption, which balances the work among the nodes and also enhances the lifetime of WSN. In case of latter more nodes were woken up to get the précised minutest details without losing the target. This is the effective lifetime that could be stressed or changed accordingly. The schedulable node set was being made in which nodes can work in their turns and the coverage and connectivity is being discussed. In order to control energy consumption as little as possible a threshold was kept for the waking up and sleeping of sensors. The simulation results show that the node scheduling brings results both in network effective lifetime and in energy consumption and this algorithm prolongs the lifetime of the network twice than PEAS.
\end{abstract}

\section{Keywords}

Node scheduling, coverage, connectivity, grid, WSN, partition.

\section{INTRODUCTION}

A wireless sensor network (WSN) consists of a set of sensing devices which collaborate to work and collect information from an area and sends it to the base station or sink. Their size varies from a shoe box to a grain. Some of the applications of wireless sensor networks are machine health care monitoring, wildlife habit study, environment, area monitoring. In case of unnatural events gas leakage, military intrusion etc, nodes track the event. WSN have been vividly used for data collection in diverse environments. While in many networks the nodes are randomly deployed in massive quantity, there is a broad range of applications advocating in support of manual deployment.

As sensor nodes are power constraint devices, longdistance transmissions should be kept to minimum in order to prolong the network lifetime and energy conservation is the prime focus for research. In ASCENT [1] nodes have four states sleep, passive, test and active they dynamically change their state to decide they will go from passive state to active and test. Idle listening uses $50-100 \%$ of the energy of that required for receiving shown by measurements [2], therefore redundant idle nodes must be turned off by using some node schedulable strategy which may prolongs network lifetime. These algorithms have operational states for nodes to balance the work between them which provides different state transitions. In PEAS [3] nodes have three states probing, working and sleeping. Firstly a node enters a probing state and checks if perception region of the node is covered or not if not so than the, node enters the working state or goes to sleeping state, and in the next round sleep nodes will be woken up . In GAF [4] nodes have three states sleeping, discovery, active. There is transition of states for the energy usage. [5] A. Mutazono et al. described a scheme of self organization sleep schedule that was based on tree frog satellite behavior. In VBS [6] there is sleep scheduling in which switching of nodes to active or sleep state, this have multiple strategies for working . [7] San-Yuan Wang et al. proposed the network in view of computational geometry and attained better coverage by very less nodes and minimized the energy utility. [8] Peyman Neamatollahi et al. proposed Clustering is an effective approach for arranging the network into a connected stream, balancing work and prolonging network lifetime. [9] Jing hui Zhang and Jun Zhang proposed local wake up scheduling, genetic algorithm based wake-up scheduling method are used for the local wake-up scheduling (LWS) strategy that could prolong the network lifetime with coverage constraint.

In node scheduling strategies the coverage of the network and its connectivity are very important. PEAS and GAF delivers connectivity and coverage based on grid and ASCENT follows a stable one.

Clustering to an extent also plays an important role for the network lifetime. An efficient manner to increase the efficiency is to arrange the network into clusters, where each cluster have a cluster head based on some decentralized and centralized clustering, a cluster head collects data from all its nodes and passes it to base station through multi hopping . Data can be fused during the data collection process. The fused data will then be transmitted to the base station directly as the data is correlated when collected through different nodes this allows the cluster heads to send data to longer distance and the cluster heads can be changed as per requirement time to time. There are different algorithms for clustering mainly like LEACH [10] which states that it is decentralized algorithm that has clusters and has two hop technology, a cluster head will be elected from the cluster nodes, and the data will be fused to make one packet as the data is correlated and less transmissions are required so it has longer lifetime than direct transmission, it is very easily implemented and is robust in nature. PEGASIS [11] It is a centralized clustering scheme in this sensor nodes are sorted and joined to form a single chain and cluster head can be elected for a cycle from this chain, node on the chain receives data from its neighbors and fuses the data with its own collected information and transmits it to the cluster head than cluster head forward the data to a base station. It is a centralized algorithm. PEGASIS is sensitive to attacks as it has a centralized processing point and also has large amount 
of overhead, which limits it. PEDAP [12] is also centralized clustering scheme and arranges the sensor nodes into a single minimum spanning tree. Only the root of the tree structure will become the cluster head. Node receives data from child nodes and it fuses the data with its own and transmits it to its parent node. At last, the fused data will reach the cluster head and being forwarded to the base station. This can reduce the path length and reduce the number of connections.

In this paper a review on node scheduling algorithm based on partition was discussed along with some information regarding clustering that is effective in enhancing the network lifetime along that coverage and connectivity was kept when the nodes would work for their respective turn. Section 2 will present the coverage and connectivity based on grid. Section 3 will introduce the scheduling algorithm .Section 4 will discuss results.

\section{COVERAGE AND CONNECTIVITY}

If some algorithm is running it should result in the desired direction and the node scheduling scheme must keep up the coverage and connectivity effectively.

\subsection{Coverage of the network:}

The scheduling scheme is applied to the nodes with overlapping. So, the monitoring area is being divided into equal grids of size $\mathrm{R} \times \mathrm{R}$ as it is difficult to identify and handle overlapping for the network .Each partition has a unique ID and partition information can be acquired through GPS. Assuming $R_{p}$ as the probing radius and value is fixed as per the hardware of the sensor. The node coverage is directly linked to node position and probing radius. In this case the values must be taken as $R \leq \sqrt{2} R_{p}$ and in worst case when the node is at vertex than it can be taken as $R \leq \sqrt{ } 2 R_{p} / 2$. Hence, the range must be given as $\sqrt{2} R_{p} / 2 \leq R \leq \sqrt{2} R_{p}$

In case complete coverage is not required than value can taken as $\mathrm{R}=\sqrt{2} \mathrm{R}_{\mathrm{p}}$.

\subsection{Connectivity of the network:}

A node called the cut point of the network, if it is turned off, there will be no connectivity. In order to keep connectivity each node or cut point must be working properly and is connected to the other.

For an extreme case, if two working nodes are at opposite vertexes $\mathrm{A}$ and $\mathrm{B}$ of the adjacent grid, they can communicate if distance $d$ is shorter to $R_{t}$ transmitting radius .So, d satisfies

$d^{2}=R^{2}+4 R^{2}$

$R$ should be given as $R \leq \sqrt{5} R_{t} / 5$

\subsection{Schedulable Node Set:}

A node is schedulable node if

- The node has a neighbor in the grid $\mathrm{A}_{\mathrm{j}}$ and it must not be a cut point.

Only schedulable nodes are scheduled independently in grid.

\section{NODE SCHEDULING ALGORITHM}

Node in this algorithm may work in two modes; they work in association to conserve energy. Usually node is in monitoring mode and In case there is working node in each grid, network monitors all grids .So, there must be a working node in each grid to save energy consumption as other nodes could be turned off.
3.1 Node Scheduling in Monitoring mode:

This further has initialization mechanism and sleep scheduling.

\subsubsection{The Initialization Mechanism}

Initially all nodes are at sleeping state. After to, nodes enter initializing state and begin to send probing message to each other in the grid $A_{j}$ in order to avoid collision $t_{\text {start }}$ (i) gets a random value $\left[0, T_{\text {init }}\right], T_{\text {init }}$ is the initialization time. In this time nodes in the grid elects the first working node, by sending probing message and partition ID is included in this probing message so that this message is received by the right node otherwise the received probing message is invalid if there exists no working node . Node enter the working state and arrange itself in the queue for the working state and if there is a working node than node communicate for the arrangement of queue and goes to sleeping state .The information is stored in first working node and is given to the next node when it wakes up.

\subsubsection{The Sleep Scheduling}

In this scheduling the working node wake up the next node in node queue one after one before it dies, as in monitoring mode one and only one node must be in working state in each grid. So, the node states are changed as per scheduling cycle. Node must not enter sleeping state while it is collecting data. $T_{\text {collect }}$ is the data acquisition cycle, time spent in collecting data is $t_{\text {collect }}$, the idle time is $t_{\text {idle }}$, the scheduling cycle is $T_{\text {schedule }}(j)$ in $A_{j}, t_{\text {work }}(j)$ is the working time , $t_{\text {sleep }}(j)$ is the sleep time , therefore

$\mathrm{T}_{\text {collect }}=\mathrm{t}_{\text {collect }}+\mathrm{t}_{\text {idle }}$

$\mathrm{T}_{\text {schedule }}(\mathrm{j})=\mathrm{t}_{\text {work }}(\mathrm{j})+\mathrm{t}_{\text {sleep }}(\mathrm{j})$

WSN works well if $T_{\text {work }}(j) \geq t_{\text {collect }}$

Node failure rate is a constant $\lambda$ and node in $\mathrm{Aj}$ and works for $\mathrm{t}_{\text {work }}(\mathrm{j})$ in scheduling cycle

$\mathrm{N}=\lambda \mathrm{t}_{\mathrm{work}}(\mathrm{j})$

Node does not fail during its working time $\mathrm{n}<1$

Therefore,

$\mathrm{t}_{\mathrm{work}}(\mathrm{j})<1 / \lambda$

Now, $\quad t_{\text {collect }} \leq t_{\text {work }}(j)<1 / \lambda$

$\mathrm{T}_{\text {schedule }}(\mathrm{j})=\mathrm{kT}_{\text {collect }}$

Where $k$ is integral multiple of the total node number $N_{j}$ in $A_{j}$ Set

$\mathrm{T}_{\text {work }}(\mathrm{j})=\mathrm{T}_{\text {collect }}$

If $\mathrm{k}=\mathrm{N}_{\mathrm{j}}$,

$\mathrm{T}_{\text {sleep }}(\mathrm{j})=\mathrm{N}_{\mathrm{j}} \mathrm{T}_{\text {collect }}-\mathrm{T}_{\text {collect }}=\left(\mathrm{N}_{\mathrm{j}}-1\right) \mathrm{t}_{\text {work }}(\mathrm{j})$

\subsection{Node Scheduling in tracking mode:}

If a moving target enters in a region the working node in that region identify it. An assisting message with partition ID information will be send by working node to other nodes in a grid, if sensor value is above threshold .Now nodes enter assisting state , after working for $\mathrm{t}_{\mathrm{work}}(\mathrm{j})$ time ,working node sends a message to the next node in the queue and it enters assisting state. The nodes in other grids come to assisting state, if target enters their perceivable range. In order to save energy in such case, the nodes will enter sleeping state if the sensor value is below threshold. 


\subsection{The Proposed algorithm:}

The main concern in WSN is the battery .As the places where the sensing is required are the remote terrains out of reach it is difficult to get there. So, the nodes are deployed through airplanes and change of battery is not an option. Therefore, such scheduling techniques must be designed that could improve the network effective lifetime by making conservation of energy for few such issues a node scheduling based on partition for WSN is effectively built.

There is different transition of the states. The nodes transit their states according to the cycle and wake up according to queue. Node enters initializing state and start the network than node will go to sleeping, working, and assisting.

\section{RESULTS}

Place Proposed algorithm was simulated in NS-2 .Randomly distributed 1000 nodes were distributed in the area of $50 \times 50$ $\mathrm{m}^{2}$. Sensor hardware parameters are similar to PEAS. The node power consumption in reception, transmission, and sleeping, idle modes are $12 \mathrm{mw}, 60 \mathrm{mw}, 0.03 \mathrm{mw}, 12 \mathrm{mw}$ respectively. The initial energy is randomly chosen in the range of 54-60 Jules .The sensing range $R_{p}$ is 3 meters, $R_{t}$ is 10 meters. Source and sink are placed in opposite corners of the field. Data report is being generated after $10 \mathrm{sec}$ and delivered to sink.

\subsection{Simulation results of Sleep Scheduling:}

Grid was built by $R=4.12 \mathrm{~m}, \mathrm{R}=2.83 \mathrm{~m}, \mathrm{R}=4.12 \mathrm{~m}$ where 4.12 $\mathrm{m}$ is approximate to $\sqrt{2} \mathrm{Rp}, 2.10 \mathrm{~m}$ is approximate to $\sqrt{2} \mathrm{Rp} / 2$. The node failure rate $\lambda=0.01$ and data acquisition cycle $\mathrm{T}_{\text {collect }}$ $=50 \mathrm{~s}$. Set $_{\text {twork }}=50 \mathrm{~s}, \mathrm{t}_{\text {sleep }}=(\mathrm{Nj}-1) \times 50 \mathrm{~s}$.

Fig.1. Depicts the dying nodes .The scale is 1000 nodes, 1 time slice $=1000 \mathrm{~s}$. Nodes completely die at 46th time slice in PEAS and proposed algorithm with $\mathrm{R}=2.10$. Last node survival time of the proposed algorithm is 65 time slice when $\mathrm{R}=2.83 \mathrm{~m}$ and 85 time slice when $\mathrm{R}=4.12 \mathrm{~m}$, better than PEAS.

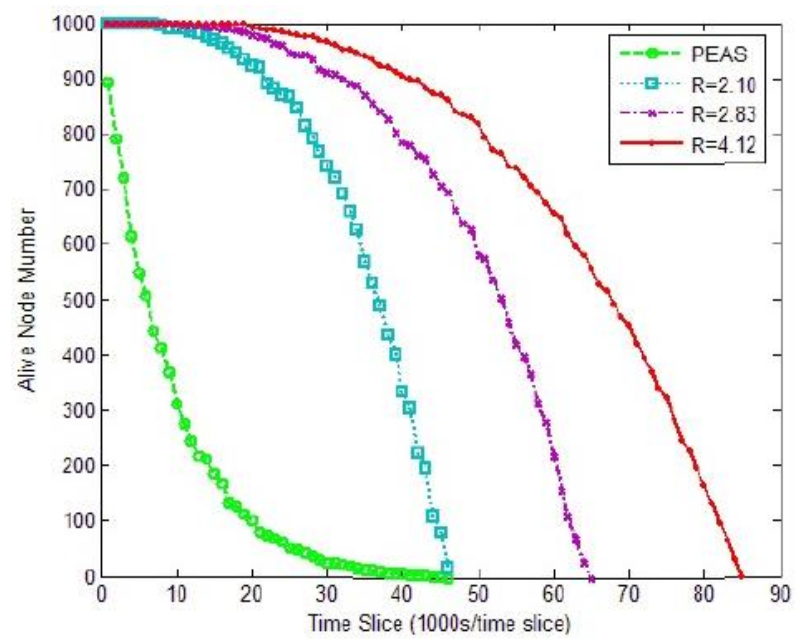

Fig 1: Node death process

Simulation results of effective lifetime when the network scale is $200,400,600,800,1000$ nodes respectively, the effective lifetime with $\mathrm{R}=2.10$ reached $35 \mathrm{TH}$ time slice, network effective lifetime when $\mathrm{R}=2.83 \mathrm{~m}$ and $\mathrm{R}=4.12 \mathrm{~m}$ are 39 time slice and 50 time slice respectively for the proposed algorithm as shown in Fig. 2.

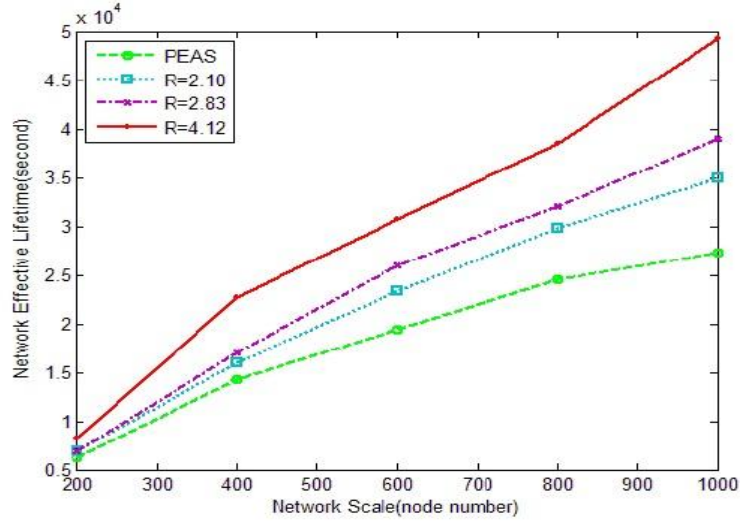

Figure 2 Network Effective Lifetime vs. Network Scale

\subsection{Simulation Results of Waking up Scheduling:}

The threshold time in tracking mode is set as 5s. Assisting nodes waked up along the target trail when the grid was built by $R=4 m$ as shown in Fig. 3 .

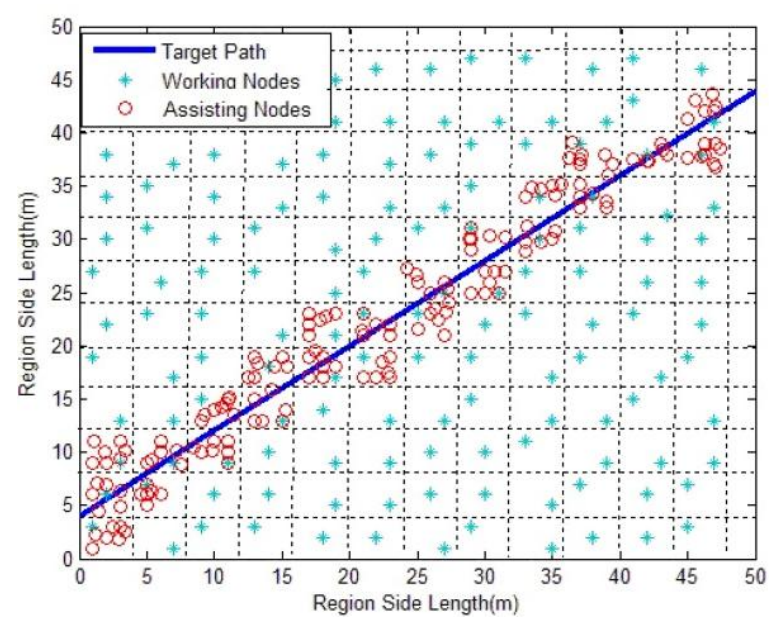

Fig 3: Nodes waked up along the target trace

Assisting nodes distribution is shown in the lower left corner of monitoring area. The working node in region of lower left corner $(0,8)$ waked up all sleeping nodes. In order to save energy assisting node must go back to sleeping time after the threshold as shown in Fig. 4.

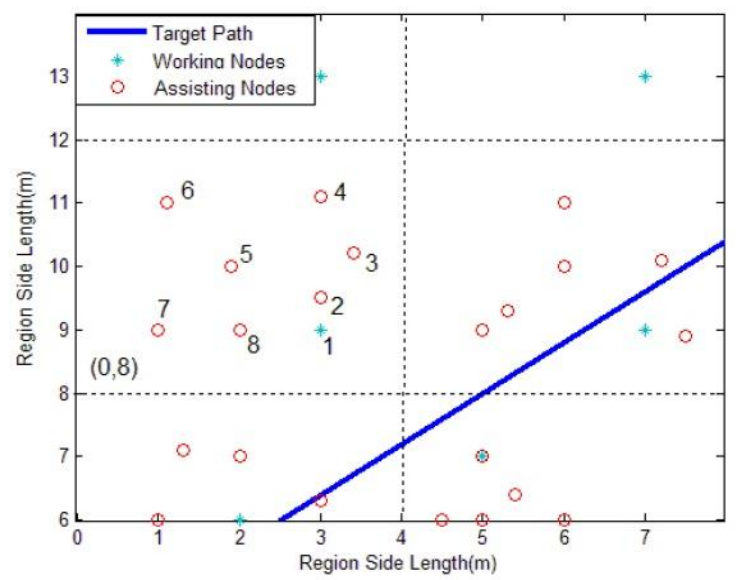

Fig 4: Assisting nodes distribution in the lower left corner of monitoring area 


\section{CONCLUSION}

Node scheduling algorithm based on partition that introduces grid was proposed.This provides network coverage and connectivity on going certainly based on partition and the redundant nodes in the monitoring mode were kept in sleeping state and in tracking mode kept in working state. Clustering may be helpful but in case consideration partition helped in achieving results better from PEAS. Results show that the effective lifetime of WSN could be extended by sleep scheduling. This can trace the targets precisely in the wake up scheduling and keeping a good observance on them. Therefore, it is an effective scheme to enhance the lifetime over other schemes and can be applied to the applications where information collection is a regular operation and tracking the event precisely is required.

\section{REFERENCES}

[1] A. Cerpa, D. Estrin, "ASCENT: adaptive selfconfiguring sensor networks topologies," IEEE Transactions on Mobile Computing, pp.272- 285, JulyAug. 2004.

[2] Wei Ye, J. Heidemann, and D. Estrin, "An energyefficient MAC protocol for wireless sensor networks," Proceedings of Twenty-First Annual Joint Conference of the IEEE Computer and Communications Societies, pp.1567-1576, 2002

[3] Fan Ye, Gary Zhong, Jesse Cheng, Songwu Lu, and Lixia Zhang, "PEAS: A Robust Energy Conserving Protocol for Long-lived Sensor Networks," Proceedings of 23rd IEEE International Conference on Distributed Computing Systems (ICDCS'03), pp. 28, 2003.

[4] Y. Xu, J. Heidemann, and D. Estrin, "Geography informed energy conservation for ad hoc routing," ACM Mobicom, 2001.

[5] A. Mutazono, M. Sugano, M. Murata, "Energy efficient sleep scheduling in wireless sensor networks inspired by satellite behavior of frogs," Proceedings of 2010 8th IEEE International Conference on Pervasive Computing and Communications Workshops (PERCOM Workshops), pp. 450-455, 29 March 2010-2 April 2010.

[6] Yaxiong Zhao, Jie Wu, Feng Li, and Sanglu Lu, "VBS: Maximum Lifetime Sleep Scheduling for Wireless
Sensor Networks Using Virtual Backbones,” Proceedings of IEEE INFOCOM 2010, pp. 1-5, 14-19 March 2010.

[7] San-Yuan Wang, Kuei-Ping Shih, Yen-Da Chen, and Hsin-Hui Ku, "Preserving Target Area Coverage in Wireless Sensor Networks by Using Computational Geometry," Proceedings of 2010 IEEE Wireless Communications and Networking Conference (WCNC), pp. 1-6, April 18-21, 2010.

[8] Peyman Neamatollahi, Hoda Taheri, Mahmoud Naghibzadeh, Mohammad-Hossein Yaghmaee, "A Hybrid Clustering Approach for ProlongingLifetime in Wireless Sensor Networks," International Symposium on Computer Networks and Distributed Systems (CNDS), pp. 170-174, February 23-24, 2011.

[9] Jing-hui Zhong and Jun Zhang, "Energy Efficient Local Wake-up Scheduling in Wireless Sensor Networks, ' IEEE Congress Evolutionary Computation (CEC), pp. 2280-2284, June 5-8, 2011.

[10] W. B. Heinzelman, A. P. Chandrakasan, and H Balakrishnan, "An Application- Specific Protocol Architecture for Wireless Microsensor Networks," IEEE Trans. Wireless Commun., vol. 1, no. 4, pp. 660-670, Oct. 2002.

[11] S. Lindsey and C. S. Raghavendra, "Pegasis: Powerefficient gathering in sensor information systems," Proc. IEEE Conf. Aerosp., Big Sky, MT, Mar. 2002, vol. 3, pp $1125-1130$

[12] H. Ö. Tan and Í. Körpeog^lu, "Power Efficient Data Gathering and Aggregation in Wireless Sensor Networks," ACM SIGMOD Record, vol. 32, no. 4, pp. 66-71, Dec. 2003.

[13] Chi-Tsun Cheng, Chi K. Tse, and Francis C. M. Lau, "A Clustering Algorithm for Wireless Sensor Networks Based on Social Insect Colonies", IEEE Sensors Journal, vol. 11, no. 3, March 2011.

[14] Bilal Abu Bakr, Leszek Lilie , "A Quantitative Comparison of Energy Consumption and WSN Lifetime for LEACH and LEACH-SM", $31^{\text {st }}$ ICDCS Workshops 2011 ,pp. 182-191 , June 20-14, 2011.

[15] Jia Xu,Ning Jin, Xizhong Lou,Ting Peng,Qian Zhou,Yanmin Chen on "Improvement of LEACH protocol for WSN" , IEEE International Conference on Fuzzy Systems and Knowledge Discovery (FSKD), pp. 2174-2177, May 29-31, 2012 